\title{
Estrutura trófica da avifauna na Reserva Estadual de Gurjaú, Zona da Mata Sul, Pernambuco, Brasil
}

\author{
Wallace R. Telino-Júnior ', Manoel M. Dias ${ }^{2}$, Severino M. de Azevedo Júnior ${ }^{3}$, \\ Rachel M. de Lyra-Neves ${ }^{1} \&$ Maria E. L. de Larrazábal ${ }^{3}$
}

\begin{abstract}
1 Unidade Acadêmica de Garanhuns, Universidade Federal Rural de Pernambuco. Rua Ernesto Dourado 390, Heliópolis, 55296-900 Garanhuns, Pernambuco,Brasil.E-mail: telinojr@ufrpe.brermlneves@ufrpe.br.

${ }^{2}$ Departamento de Ecologia e Biologia Evolutiva, Universidade Federal de São Carlos. Caixa Postal 676, 13565-905 São Carlos, São Paulo, Brasil. E-mail: manoelmd@power.ufscar.br.

${ }^{3}$ Mestrado em Biologia Animal, Departamento de Zoologia, Universidade Federal de Pernambuco. Avenida Professor Moraes Rego 1235, Cidade Universitária, 50670-420 Recife, Pernambuco, Brasil. E-mail: smaj@ufpe.br; mells@ufpe.br.
\end{abstract}

\begin{abstract}
Trophic structure of bird community of Reserva Estadual de Gurjaú, Zona da Mata Sul, Pernambuco State, Brazil. The study of trophic structure of birds community from Gurjaú Estadual Reserve $\left(8^{\circ} 14^{\prime} \mathrm{S}, 35^{\circ} \mathrm{O} 3^{\prime} \mathrm{W}\right)$ has involved 225 bird species, from that 220 registered by observation and capture, four by interview with native people from surrounding area and one in the ornithological collection of Universidade Federal de Pernambuco. The groups with the higher number of species were Tyrannidae $(\mathrm{N}=43)$ and Emberizidae $(N=36)$. Concerning the guilds, insectivorous $(N=99)$ and omnivorous $(N=59)$ were the most representative among the especies.
\end{abstract}

KEY WORDS. Atlantic Rain Forest, bird ecology.

RESUMO. O estudo da estrutura trófica da avifauna da Reserva Estadual de Gurjaú ( $8^{\circ} 14^{\prime} \mathrm{S}, 35^{\circ} 03^{\prime} \mathrm{W}$ ) envolveu 225 espécies de aves identificadas, das quais, 220 registradas por meio de observações in situ e capturas, quatro por meio de entrevistas com moradores da área do entorno e uma através de consulta à Coleção Ornitológica da Universidade Federal de Pernambuco. As famílias que se destacaram com o maior número de espécies foram Tyrannidae $(\mathrm{N}=43)$ e Emberizidae $(\mathrm{N}=36)$. Dentre as guildas, os insetívoros $(\mathrm{N}=99)$ e onívoros $(\mathrm{N}=59)$ englobaram a maioria das espécies.

PALAVRAS CHAVE. Ecologia de aves, Floresta Atlântica.

A Floresta Atlântica compreende cerca de $1.300 .000 \mathrm{Km}^{2}$, estendendo-se por quatro das cinco regiões geográficas brasileiras; atualmente está reduzida a cerca de $7,6 \%$ da sua cobertura vegetal original (Dossiê Mata AtLÂNTICA 2001). Possuem elevado endemismo para muitos grupos taxonômicos, particularmente aves e plantas (Aleixo \& GaletTi 1997, Silva \& TABARelli 2000).

A maior parte das áreas remanescentes encontra-se protegida em Unidades de Conservação ou sob pressão de atividades rurais ou expansões urbanas (Dossiê MATA ATLÂNTICA 2001). Além da grande devastação desde a época da colonização, a Mata Atlântica continua sendo gradativamente destruída e atualmente a perda total é superior a 70\% de sua vegetação nativa (Myers et al. 2000, Brooks et al. 2002).

O Nordeste do Brasil é o setor mais ameaçado da Mata Atlântica em conseqüência dos desflorestamentos, restando apenas $2 \%$ de sua área original (Silva \& Tabarelli 2000). Ao estudarem a Zona da Mata Sul do Estado de Pernambuco, RANTA et al. (1998), verificaram que 48\% dos fragmentos são menores que 10 ha e somente $7 \%$ deles são maiores que 100 ha.

No estado de Pernambuco a cultura da cana-de-açúcar foi a principal causa do desmatamento, resultando na fragmentação de áreas naturais. O que ameaça a permanência das espécies, tanto pela perda de hábitat quanto pela ampliação do efeito de borda para o interior dos fragmentos. Com isso, as conseqüências são bastante negativas, não apenas sobre as aves dependentes do interior de floresta, mas também sobre outras populações, como por exemplo, as participantes de bandos mistos típicos de regiões de borda, as quais, têm a estrutura dos grupos sociais alteradas (MARINI 2000, PRIMACK \& Rodrigues 2001).

A estrutura da vegetação (altura, estratificação vertical e heterogeneidade) constitui um dos fatores preponderantes na determinação do número de espécies de aves em nível local (Karr \& Roth 1971, Terborgh 1984, Aleixo 1999, Marini 2000). Um aspecto importante é o estudo da alimentação das aves 
que sobrevivem nestas áreas, que pode fornecer informações sobre a estrutura trófica de comunidades, bem como das condições físicas do ambiente (Piratelli \& Pereira 2002).

As aves são um dos grupos animais mais distintos e bem estudados, podendo ser utilizados como bio-indicadoras de alterações ambientais (VERNER 1981). O conhecimento das exigências ecológicas de muitas famílias, gêneros e espécies de aves pode ser suficiente em diversas situações para indicar condições ambientais às quais são sensíveis; portanto, alterações de vegetação implicam que o ambiente natural pode tornar-se impróprio para abrigar aves que exigem condições específicas para sobreviver (DonATELli et al. 2004).

A estrutura e composição de uma comunidade de aves sofrem mudanças quando ocorre alguma alteração na vegetação, seja ela natural ou provocada por atividades humanas (Aleixo 1999). Além do mais, estudos sobre a estrutura trófica de comunidades de aves podem revelar que perturbações ambientais levam a um aumento no número de espécies onívoras e insetívoras menos especializadas e diminuição de frugívoros (MOTTA-JÚNIOR 1990).

Inventários avifaunísticos já foram realizados em diversos fragmentos de Mata Atlântica no estado de Pernambuco. Todavia, as informações sobre a estrutura trófica das comunidades de aves encontradas nesses fragmentos são escassas. Desta forma, o objetivo deste estudo foi verificar a estrutura trófica da avifauna da avifauna da Reserva Ecológica de Gurjaú.

\section{MATERIAL E MÉTODOS}

A Reserva Estadual de Gurjaú (RESG) está localizada nos municípios do Cabo de Santo Agostinho, Moreno e Jaboatão dos Guararapes, Zona da Mata, sul do Estado de Pernambuco

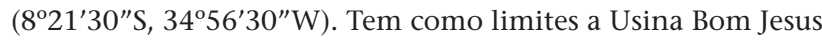
e os Engenhos São Salvador e Espírito. Está inserida em uma única propriedade pública, pertencente à Companhia Pernambucana de Abastecimento de Água (COMPESA), onde existe uma estação de tratamento. No interior da reserva encontram-se três açudes: Sucupema, Gurjaú e São Salvador (FIDEM 1987, Lyra-Neves et al. 2004).

Possui uma área total de 1.077,10 ha, distribuídos segundo a Fidem (1987) em: 744,47 ha (Cabo de Santo Agostinho); 175,19 ha (Moreno) e 157,44 ha (Jaboatão dos Guararapes). Foi definida como Reserva Estadual pela Lei Estadual $n^{\circ} 9.989$ em 1987. Apesar de ser um remanescente de Mata Atlântica, a sua cobertura vegetal não é uniforme, apresentando trechos de vegetação densa e outros bastante alterados em sua estrutura e fisionomia e por estarem completamente ilhados por cana-deaçúcar e outras culturas, principalmente de subsistência (FIDEM 1987, Lyra-Neves et al. 2004). A RESG encontra-se ameaçada pelos canaviais, devido ao uso de agrotóxicos e herbicidas, além, do uso indiscriminado pelos posseiros, os quais retiram madeira para construção e reformas de suas casas e para a queima em fogões a lenha e ainda, pode-se destacar a caça de animais silvestres (Lyra-Neves et al. 2004).
As comunidades de aves da Reserva Ecológica de Gurjaú foram estudadas no período de agosto de 2002 a abril de 2003, compreendendo as estações seca e chuvosa, com expedições mensais de cinco dias cada, totalizando 45 dias de atividades de campo e um esforço amostral de 360 horas.

As informações sobre a avifauna foram obtidas através da observação direta dos indivíduos com auxílio de binóculos, onde, o pesquisador percorria uma trilha existente na mata, percorrendo o interior, a borda da mata e áreas abertas. Durante as caminhadas foram anotados os seguintes dados: visualizações e/ou vocalizações, estrato ocupado, número de indivíduos (exemplares isolados, em pares ou em grupos). Também se utilizou a identificação por meio das vocalizações e "play-back" com o auxílio de um minicassete Aiwa TP560 e gravador Sony TCM 5000, microfone Leson ML8 e através das capturas com redes de neblina. Para as atividades de captura, foram utilizadas 12 redes de neblina $(2,5 \times 12 \mathrm{~m}$ e $36 \mathrm{~mm}$ de malha) dispostas em dupla nos dois períodos do dia. Pela manhã, as redes eram abertas às 5:00 h e fechadas às 11:00 h e no período da tarde eram abertas das 15:00 às 17:00 h. Ainda, foram realizadas visitas à coleção ornitológica da Universidade Federal de Pernambuco para identificação e contagem de exemplares coletados na RESG e ali depositados. Também, com uma visão conservacionista por meio do trabalho de biologia da conservação, aplicou-se questionário para a população da RESG, onde alguns itens tratavam sobre os registros da avifauna local questionando a presença e ausências das espécies conhecidas pelos moradores da região.

Os Registros das aves foram categorizados como: (OB) observação visual, (V) vocalização, (CP) captura com rede de neblina (malha de $36 \mathrm{~mm}$ ), (CL) Coleção e (EM) entrevistas.

As dietas foram determinadas através dos registros de campo e bibliografia específica (e.g. Moojen et al.1941, Hempel 1949, Kuhlmann \& Jimbo 1957, Jimbo 1957, Schubart et al. 1965, Willis 1979, Motta-Júnior 1990, Terborgh et al.1990, Sick 1997). As categorias tróficas consideradas foram: Carnívoro (C), Detritívoro (D), Frugívoro (F), Granívoro (G), Insetívoro (I), Nectarívoro (N), Onívoro (O) e Piscívoro (P).

A nomenclatura científica utilizada é aquela proposta por SICK (1997), todavia, foram acatadas as resoluções do Comitê Brasileiro de Registros Ornitológicos (CBRO). Os nomes vulgares de acordo com Sick (1997) e Farias et al. (2000).

\section{RESULTADOS E DISCUSSÃO}

Foram registradas 225 espécies de aves na Reserva Estadual de Gurjaú, das quais, 220 durante as observações e capturas, quatro foram citadas nas entrevistas com os moradores da região: Ortalis gutatta Spix, 1825, Nyctibius griseus (Gmelin, 1789), Sicalis luteola (Sparrman, 1789) e Sporophila albogularis (Spix, 1825) e uma tombada na coleção científica da Universidade Federal de Pernambuco, Momotus momota (Linnaeus, 1766). As 225 espécies registradas neste trabalho estão distribuídas em 40 famílias e 12 subfamílias o que corresponde a 
45,18\% da avifauna de Pernambuco (498 espécies) (Tab. I). A riqueza de espécies encontrada na RESG se assemelha àquela citada por Motta-Júnior (1990), D’Angelo-Neto et al. (1998), Anjos (1998), Aleixo (1999), Anjos \& Boçon (1999), Dário \& Almeida (2000), Krügel \& Anjos (2000), Anjos (2001), Efe et al. (2001), Guilherme (2001), Blamires et al. (2001), SANTos (2001) e Silveira et al. (2003), em levantamentos semelhantes ao realizado nesta pesquisa.

Dentre as famílias mais representativas, registradas na RESG, Tyrannidae e Emberizidae destacaram-se por possuírem o maior número de espécies (43 e 36 espécies respectivamente) (Fig. 1). Essas espécies provavelmente seriam mais tolerantes a ambientes alterados pelo homem, pois resultados semelhantes também foram encontrados por MotTa-Júnior (1990), Anjos \& Bóçon (1999), Krưgel \& Anjos (2000), Anjos (2001), Efe et al. (2001), GuilHerme (2001).

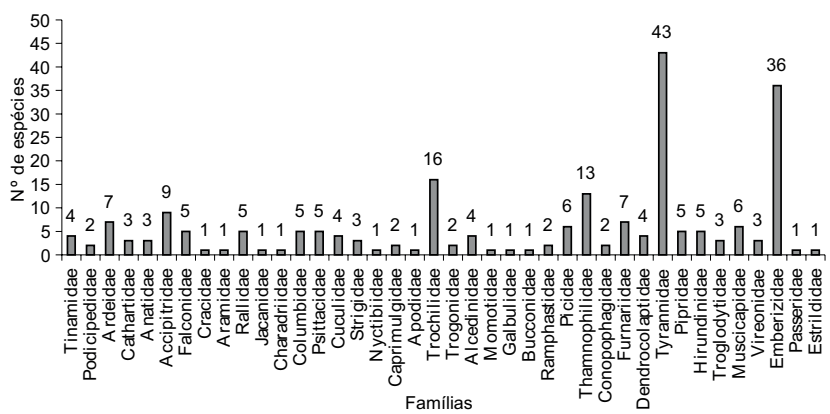

Figura 1. Número de espécies por famílias encontradas durante os censos captura e entrevistas, na Reserva Estadual de Gurjaú, Cabo de Santo Agostinho, Pernambuco.

A estrutura trófica da avifauna da RESG demonstrou um predomínio das espécies insetívoras seguidas por onívoras (Tabs I e II). Dados semelhantes foram constatados por Willis (1979), MotTa-Júnior (1990), Anjos (1998, 2001), Aleixo (1999), Anjos \& Bóçon (1999), Dário \& Almeida (2000), Krügel \& Anjos (2000), Blamires et al. (2001), SANTos (2001), Silveira et al. (2003), diferindo apenas do resultado encontrado por EFE (2001), o qual, verificou uma presença maior de onívoros em relação aos insetívoros. A variação nessas duas categorias pode ser explicada por um aumento na onivoria em áreas mais antropizadas, como fragmentos de mata, assim como em áreas cobertas por vegetação secundária ou exótica; a onivoria teria um efeito tampão contra flutuações no suprimento de alimentos (WILLIS 1976, D’Angelo-Neto et al. 1998, Blamires et al. 2001). Entretanto, ALMEIDA (1982) comenta que o número de espécies onívoras em geral é maior nas matas menos alteradas e o de insetívoros aumenta nas mais alteradas sendo este fato observado para o fragmento de Gurjaú. WiLlis (1979) e Anjos (1998) citam que aves insetívoras escaladoras de tronco e galho (pica-paus e arapaçus) são localmente extintas mais rapidamente com a diminuição da área de forrageamento. Aleixo \& Vielliard (1995) indicaram os insetívoros como o grupo de espécies mais sensível à fragmentação florestal.

As aves de dietas mais especializadas como as frugívoras aparecem em terceiro lugar, onde se destacam aquelas de maior porte, a exemplo de Crypturellus soui (Hermann, 1783), Pionus maximiliani (Kuhl, 1820), Pteroglossus aracari (Linnaeus, 1758) e Pteroglossus inscriptus Swainson, 1822, ocorrendo em todas as áreas percorridas durante a pesquisa. Estas aves, também, foram registradas para fragmentos de Mata Atlântica nos estados de Pernambuco e Alagoas por Pinto (1940), Pacheco \& Whitney (1995) e Silveira et al. (2003).

O percentual de espécies frugívoras indica que na reserva de Gurjaú ainda existem plantas frutíferas que contribuem para a manutenção dessas aves, principalmente dos frugívoros de grande porte. Além das espécies de árvores nativas, outras espécies (e.g. bananeira) são cultivadas por moradores do entorno, e fornecem alimento para pequenos frugívoros como: Tangara fastuosa (Lesson, 1831), T. cyanocephala (Müller, 1766) e T. velia (Wied, 1830), durante a escassez de recursos nativos.

Em Gurjaú foi observado que papagaios, araçaris, surucuás e traupídeos realizavam deslocamentos entre os fragmentos da reserva. De acordo com Willis (1979), Anjos (1998) e GUILHERME (2001) os frugívoros apresentam maior capacidade de deslocamento, podendo procurar alimento em outros fragmentos desde que não estejam muito distantes.

Os granívoros estão bem representados por espécies das famílias Columbidae e Emberizidae encontradas forrageando nas áreas de interior de mata, borda e áreas abertas. Os dados sugerem que o entorno dos fragmentos florestais, bem como as clareiras são compostas por gramíneas e contribuem para uma presença maior de alguns columbídeos: Columbina passerina (Linnaeus, 1758), Columbina minuta (Linnaeus, 1766) e Columbina talpacoti (Temminck, 1811) e emberizideos: Volatinia jacarina (Linnaeus, 1766), Sporophila spp., Tiaris fuliginosus (Wied, 1831) e Paroaria dominicana (Linnaeus, 1758) (Tab. I). Anjos (1998) discute que com a fragmentação e conseqüentemente o aumento da área de borda os granívoros são beneficiados por utilizarem essas áreas para forrageio.

$\mathrm{Na}$ escala de representatividade, as aves nectarívoras representadas pelos Trochilidae, possuem espécies que são comumente encontradas em áreas de capoeira e no interior de fragmentos florestais (Jonhs 1991, STOUfFer \& BierRegaARd 1995b, Guilherme 2001). Na RESG, foram identificadas 16 espécies desta família (Tab. I), as quais são eficientes em explorar áreas de vegetação secundária onde existam flores nativas ou exóticas disponíveis ao longo do ano (Guilherme 2001).

As aves carnívoras foram representadas pelos Accipitridae, Falconidae e Strigidae. São consideradas espécies de topo de cadeia trófica (Efe 2001) e algumas espécies destacaram-se sendo consideradas residentes, a exemplo de Elanus leucurus (Vieillot, 1818), Leptodon cayanensis (Latham, 1790), Rupornis magnirostris (Gmelin, 1789), Megascops choliba (Vieillot, 1817) 
Tabela I. Avifauna registrada para a Reserva Ecológica de Gurjaú, zona da mata sul de Pernambuco, no período de agosto de 2002 a abril de 2003. Registro: (OB) observação visual, (V) vocalização, (CP) captura em rede de neblina, (CL) Coleção, (E) entrevista; Dieta: (C) carnívoro, (D) detritívoro, (F) frugívoro, (G) granívoro, (I) insetívoro, $(\mathrm{N})$ nectarívoro, $(\mathrm{O})$ onívoro, $(\mathrm{P})$ piscívoro.

\begin{tabular}{|c|c|c|c|}
\hline Família/Espécie & Nome popular & Registro & Dieta \\
\hline \multicolumn{4}{|l|}{ Tinamidae } \\
\hline Crypturellus soui (Hermann, 1783) & Nhambu-mata-cachorro & V & $\mathrm{F}$ \\
\hline Crypturellus parvirostris (Wagler, 1827) & Nhambu-espanta-boiada & V & $\mathrm{O}$ \\
\hline Crypturellus tataupa (Temminck, 1815) & Nhambu-de-pé-roxo & V & G \\
\hline Nothura boraquira (Spix, 1825) & Codorna-do-nordeste & $\mathrm{OB}, \mathrm{V}$ & I \\
\hline \multicolumn{4}{|l|}{ Podicipedidae } \\
\hline Tachybaptus dominicus (Linnaeus, 1766) & Mergulhão-pequeno & $\mathrm{OB}$ & $\mathrm{O}$ \\
\hline Podilymbus podiceps (Linnaeus, 1758) & Mergulhão-caçador & $\mathrm{OB}$ & $\mathrm{O}$ \\
\hline \multicolumn{4}{|l|}{ Ardeidae } \\
\hline Ardea alba Linnaeus, 1758 & Garça-branca-grande & $\mathrm{OB}$ & $\mathrm{O}$ \\
\hline Egretta thula (Molina, 1782) & Garça-branca-pequena & $\mathrm{OB}$ & $\mathrm{O}$ \\
\hline Bubulcus ibis (Linnaeus, 1758) & Garça-vaqueira & $\mathrm{OB}$ & $\mathrm{O}$ \\
\hline Butorides striata (Linnaeus, 1758) & Socozinho & $\mathrm{OB}$ & $\mathrm{O}$ \\
\hline Tigrisoma lineatum (Boddaert, 1783) & Socó-boi & $\mathrm{OB}$ & $\mathrm{O}$ \\
\hline Ixobrychus exilis (Gmelin, 1789) & Socó-vermelho & $\mathrm{OB}$ & $\mathrm{O}$ \\
\hline Botaurus pinnatus (Wagler, 1829) & Socó-boi-baio & $\mathrm{OB}$ & $\mathrm{O}$ \\
\hline \multicolumn{4}{|l|}{ Cathartidae } \\
\hline Coragyps atratus (Bechstein, 1793) & Urubu-de-cabeça-preta & $\mathrm{OB}$ & $\mathrm{D}$ \\
\hline Cathartes aura Linnaeus, 1758 & Urubu-de-cabeça-vermelha & $\mathrm{OB}$ & $\mathrm{D}$ \\
\hline Cathartes burrovianus Cassin, 1845 & Urubu-de-cabeça-amarela & $\mathrm{OB}$ & $\mathrm{D}$ \\
\hline \multicolumn{4}{|l|}{ Anatidae } \\
\hline Dendrocygna viduata (Linnaeus, 1766) & Marreca-irerê & $\mathrm{OB}, \mathrm{V}$ & $\mathrm{O}$ \\
\hline Amazonetta brasiliensis (Gmelin, 1789) & Patarrona & $\mathrm{OB}$ & $\mathrm{O}$ \\
\hline Nomonyx dominica (Linnaeus, 1766) & Bico-roxo & $\mathrm{OB}$ & $\mathrm{O}$ \\
\hline \multicolumn{4}{|l|}{ Accipitridae } \\
\hline Elanus leucurus (Vieillot, 1818) & Gavião-peneira & $\mathrm{OB}$ & $\mathrm{C}$ \\
\hline Gampsonyx swainsonii Vigors, 1825 & Gaviãozinho & $\mathrm{OB}$ & 1 \\
\hline Leptodon cayanensis (Latham, 1790) & Gavião-de-cabeça-cinza & $\mathrm{OB}$ & I \\
\hline Buteo albicaudatus Vieillot, 1816 & Gavião-de-cauda-branca & $\mathrm{OB}$ & I \\
\hline Buteo nitidus (Latham, 1790) & Gavião-pedrez & $\mathrm{OB}$ & I \\
\hline Buteo brachyurus Vieillot, 1816 & Gavião-de-cauda-curta & $\mathrm{OB}$ & I \\
\hline Rupornis magnirostris (Gmelin, 1788) & Gavião-carijó & $\mathrm{OB}, \mathrm{V}$ & $\mathrm{C}$ \\
\hline Buteogallus urubitinga (Gmelin, 1788) & Gavião-preto & $\mathrm{OB}, \mathrm{V}$ & 1 \\
\hline Spizaetus tyrannus (Wied, 1820) & Gavião-pega-macaco & $\mathrm{OB}, \mathrm{V}$ & C \\
\hline \multicolumn{4}{|l|}{ Falconidae } \\
\hline Herpetotheres cachinans Linnaeus, 1758 & Gavião-acauã & $\mathrm{OB}, \mathrm{V}$ & $\mathrm{C}$ \\
\hline Micrastur semitorquatus (Vieillot, 1817) & Gavião-da-mata & $\mathrm{OB}$ & $\mathrm{C}$ \\
\hline Micrastur gilvicollis (Vieillot, 1817) & Gavião-mateiro & $\mathrm{OB}$ & $\mathrm{C}$ \\
\hline Milvago chimachima (Vieillot, 1816) & Gavião-carrapateiro & $\mathrm{OB}$ & $\mathrm{C}$ \\
\hline Caracara plancus (Miller, 1777) & Gavião-carcará & $\mathrm{OB}$ & $\mathrm{O}$ \\
\hline \multicolumn{4}{|l|}{ Cracidae } \\
\hline Ortalis guttata (Spix, 1825) & Aracuã & EN & $\mathrm{F}$ \\
\hline
\end{tabular}


Tabela I. Avifauna registrada para a Reserva Ecológica de Gurjaú, zona da mata sul de Pernambuco, no período de agosto de 2002 a abril de 2003. Registro: (OB) observação visual, (V) vocalização, (CP) captura em rede de neblina, (CL) Coleção, (E) entrevista; Dieta: (C) carnívoro, (D) detritívoro, (F) frugívoro, (G) granívoro, (I) insetívoro, $(\mathrm{N})$ nectarívoro, $(\mathrm{O})$ onívoro, (P) piscívoro.

\begin{tabular}{|c|c|c|c|}
\hline Família/Espécie & Nome popular & Registro & Dieta \\
\hline \multicolumn{4}{|l|}{ Aramidae } \\
\hline Aramus guarauna (Linnaeus, 1766) & Carão & $\mathrm{OB}$ & C \\
\hline \multicolumn{4}{|l|}{ Rallidae } \\
\hline Aramides cajanea (Müller, 1776) & Três-cocos & $\mathrm{V}$ & $\mathrm{O}$ \\
\hline Porzana albicollis (Vieillot, 1819) & Sanã-carijo & $\mathrm{OB}$ & $\mathrm{O}$ \\
\hline Laterallus viridis (Müller, 1776) & Siricóia-mirim & $\mathrm{OB}, \mathrm{V}$ & $\mathrm{O}$ \\
\hline Gallinula chloropus (Linnaeus, 1758) & Galinha-d'água-preta & $\mathrm{OB}, \mathrm{V}$ & $\mathrm{O}$ \\
\hline Porphyrio martinica (Linnaeus, 1766) & Galinha-d'água-azul & $\mathrm{OB}, \mathrm{V}$ & $\mathrm{O}$ \\
\hline \multicolumn{4}{|l|}{ Jacanidae } \\
\hline Jacana jacana (Linnaeus, 1766) & Jaçanã & $\mathrm{OB}, \mathrm{V}$ & $\mathrm{O}$ \\
\hline \multicolumn{4}{|l|}{ Charadriidae } \\
\hline Vanellus chilensis (Molina, 1782) & Tetéu & $\mathrm{OB}, \mathrm{V}$ & $\mathrm{O}$ \\
\hline \multicolumn{4}{|l|}{ Columbidae } \\
\hline Columbina passerina (Linnaeus, 1758) & Rolinha-cinzenta & OB & G \\
\hline Columbina minuta (Linnaeus, 1766) & Rolinha-cafofa & OB & G \\
\hline Columbina talpacoti (Temminck, 1811) & Rolinha-caldo-de-feijão & $\mathrm{OB}$ & G \\
\hline Leptotila rufaxilla (Richard \& Bernard, 1792) & Juriti-gemedeira & $\mathrm{OB}, \mathrm{V}, \mathrm{CP}$ & G \\
\hline Geotrygon montana (Linnaeus, 1758) & Juriti-vermelha & $\mathrm{OB}, \mathrm{V}, \mathrm{CP}$ & G \\
\hline \multicolumn{4}{|l|}{ Psittacidae } \\
\hline Aratinga leucophthalma (Statius Muller, 1776) & Periquitão-maracanã & $\mathrm{OB}, \mathrm{V}$ & $\mathrm{F}$ \\
\hline Aratinga cactorum (Kuhl, 1820) & Jandaia-gangarra & $\mathrm{OB}, \mathrm{V}$ & $\mathrm{F}$ \\
\hline Forpus xanthopterygius (Spix, 1824) & Tuim & $\mathrm{OB}, \mathrm{V}$ & $\mathrm{F}$ \\
\hline Touit surdus (Kuhl, 1820) & Apuim-de-cauda-amarela & $\mathrm{OB}, \mathrm{V}$ & $\mathrm{F}$ \\
\hline Pionus maximiliani (Kuhl, 1820) & Maitaca-de-maximiliano & $\mathrm{OB}, \mathrm{V}$ & $\mathrm{F}$ \\
\hline \multicolumn{4}{|l|}{ Cuculidae } \\
\hline Piaya cayana (Linnaeus, 1766) & Alma-de-gato & $\mathrm{OB}, \mathrm{V}$ & I \\
\hline Crotophaga ani Linnaeus, 1758 & Anu-preto & $\mathrm{OB}, \mathrm{V}$ & I \\
\hline Guira guira (Gmelin, 1788) & Anu-branco & $\mathrm{OB}, \mathrm{V}$ & I \\
\hline Tapera naevia (Linnaeus, 1766) & Peitica & $\mathrm{OB}, \mathrm{V}$ & I \\
\hline \multicolumn{4}{|l|}{ Strigidae } \\
\hline Megascops choliba (Vieillot, 1817) & Corujinha-do-mato & $\mathrm{V}$ & 1 \\
\hline Pulsatrix perspicillata (Latham, 1790) & Coruja-murucututu & $\mathrm{OB}, \mathrm{V}$ & $\mathrm{C}$ \\
\hline Glaucidium brasilianum (Gmelin, 1788) & Caburé-ferrugem & V & $\mathrm{C}$ \\
\hline \multicolumn{4}{|l|}{ Nyctibiidae } \\
\hline Nyctibius griseus (Gmlin, 1789) & Mãe-da-lua & EN & I \\
\hline \multicolumn{4}{|l|}{ Caprimulgidae } \\
\hline Nyctidromus albicollis (Gmelin, 1789) & Bacurau & $\mathrm{OB}, \mathrm{V}$ & I \\
\hline Hydropsalis torquata (Gmelin, 1789) & Bacurau-tesoura & $\mathrm{OB}, \mathrm{V}$ & 1 \\
\hline \multicolumn{4}{|l|}{ Apodidae } \\
\hline Tachornis squamata (Cassin, 1853) & Tesourinha & $\mathrm{OB}$ & 1 \\
\hline \multicolumn{4}{|l|}{ Trochilidae } \\
\hline Glaucis hirsutus (Gmelin, 1788) & Beija-flor-balança-rabo-de-bico-torto & $\mathrm{OB}, \mathrm{V}, \mathrm{CP}$ & $N$ \\
\hline Phaethornis pretrei (Lesson \& Delattre, 1839) & Beija-flor-rabo-branco-de-sobre-amarelo & $\mathrm{OB}, \mathrm{V}, \mathrm{CP}$ & $\mathrm{N}$ \\
\hline & & & Contin \\
\hline
\end{tabular}


Tabela I. Continuação.

\begin{tabular}{|c|c|c|c|}
\hline Família/Espécie & Nome popular & Registro & Dieta \\
\hline Phaethornis ruber (Linnaeus, 1758) & Beija-flor-besourinho-da-mata & $\mathrm{OB}, \mathrm{V}, \mathrm{CP}$ & $\mathrm{N}$ \\
\hline Eupetomena macroura (Gmelin, 1788) & Beija-flor-rabo-de-tesoura & $\mathrm{OB}, \mathrm{V}$ & $\mathrm{N}$ \\
\hline Florisuga fusca (Vieillot, 1817) & Beija-flor-de-rabo-preto-e-branco & $\mathrm{OB}, \mathrm{V}$ & $\mathrm{N}$ \\
\hline Anthracothorax nigricollis (Boddaert, 1783) & Bizunga & $\mathrm{OB}, \mathrm{V}$ & $\mathrm{N}$ \\
\hline Chrysolampis mosquitus (Linnaeus, 1758) & Beija-flor-vermelho & $\mathrm{OB}, \mathrm{V}$ & $\mathrm{N}$ \\
\hline Chlorestes notata (Reich, 1793) & Beija-flor-de-garganta-azul & $\mathrm{OB}, \mathrm{V}$ & $\mathrm{N}$ \\
\hline Chlorostilbon aureoventris (d'Orbigny \& Lafresnaye, 1838) & Beija-flor-besourinho-de-bico-vermelho & $\mathrm{OB}, \mathrm{V}$ & $\mathrm{N}$ \\
\hline Thalurania watertonii (Bourcier, 1847) & Beija-flor-de-costa-violeta & $\mathrm{OB}, \mathrm{V}$ & $\mathrm{N}$ \\
\hline Hylocharis cyanus (Vieillot, 1818) & Beija-flor-roxo & $\mathrm{OB}, \mathrm{V}$ & $\mathrm{N}$ \\
\hline Amazilia versicolor (Vieillot, 1818) & Beija-flor-de-banda-branca & $\mathrm{OB}, \mathrm{V}$ & $\mathrm{N}$ \\
\hline Amazilia fimbriata (Gmelin, 1788) & Beija-flor-de-garganta-verde & $\mathrm{OB}, \mathrm{V}$ & $\mathrm{N}$ \\
\hline Amazilia leucogaster (Gmelin, 1788) & Beija-flor-de-barriga-branca & $\mathrm{OB}, \mathrm{V}$ & $\mathrm{N}$ \\
\hline Heliothryx auritus (Gmelin, 1788) & Beija-flor-de-bochecha-azul & $\mathrm{OB}, \mathrm{V}$ & $\mathrm{N}$ \\
\hline Heliactin bilophus (Temminck, 1820) & Beija-flor-chifre-de-ouro & $\mathrm{OB}, \mathrm{V}$ & $\mathrm{N}$ \\
\hline \multicolumn{4}{|l|}{ Trogonidae } \\
\hline Trogon viridis Linnaeus, 1766 & Surucuá-de-barriga-amarela & $\mathrm{V}$ & $\mathrm{O}$ \\
\hline Trogon curucui Linnaeus, 1766 & Surucuá-de-coroa-azul & $\mathrm{OB}, \mathrm{V}$ & $\mathrm{O}$ \\
\hline \multicolumn{4}{|l|}{ Alcedinidae } \\
\hline Ceryle torquata (Linnaeus, 1766) & Martim-pescador-grande & $\mathrm{O}$ & $P$ \\
\hline Chloroceryle amazona (Latham, 1790) & Martim-pescador-verde & $\mathrm{O}$ & $P$ \\
\hline Chloroceryle americana (Gmelin, 1788) & Martim-pescador-pequeno & $\mathrm{O}$ & $P$ \\
\hline Chloroceryle aenea (Pallas, 1764) & Martim-pescador-anão & $\mathrm{O}$ & $P$ \\
\hline \multicolumn{4}{|l|}{ Momotidae } \\
\hline Momotus momota (Linnaeus, 1766) & Udu-de-coroa-azul & $\mathrm{CL}$ & 1 \\
\hline \multicolumn{4}{|l|}{ Galbulidae } \\
\hline Galbula ruficauda Curvier, 1816 & Bico-de-agulha & $\mathrm{OB}, \mathrm{V}$ & 1 \\
\hline \multicolumn{4}{|l|}{ Bucconidae } \\
\hline Nystalus maculatus (Gmelin, 1788) & Dorminhoco & $\mathrm{OB}, \mathrm{V}$ & 1 \\
\hline \multicolumn{4}{|l|}{ Ramphastidae } \\
\hline Pteroglossus aracari (Linnaeus, 1758) & Araçari-de-bico-branco & $\mathrm{OB}, \mathrm{V}$ & $\mathrm{F}$ \\
\hline Pteroglossus inscriptus Swainson, 1822 & Araçari-miúdo-de-bico-riscado & $\mathrm{OB}, \mathrm{V}$ & $\mathrm{F}$ \\
\hline \multicolumn{4}{|l|}{ Picidae } \\
\hline Picumnus cirratus Temminck, 1825 & Pica-pau-anão-barrado & $\mathrm{OB}, \mathrm{V}$ & 1 \\
\hline Picumnus exilis Lichtenstein, 1823 & Pica-pau-anão-dourado & $\mathrm{OB}, \mathrm{V}$ & I \\
\hline Picumnus fulvescens Stager, 1961 & Pica-pau-anão-de-pernambuco & $\mathrm{OB}, \mathrm{V}$ & 1 \\
\hline Dryocopus lineatus (Angs \& Penard, 1918) & Pica-pau-de-banda-branca & $\mathrm{OB}, \mathrm{V}$ & 1 \\
\hline Veniliornis passerinus (Linnaeus, 1766) & Pica-pau-pequeno & $\mathrm{OB}, \mathrm{V}, \mathrm{CP}$ & I \\
\hline Veniliornis affinis (Swainson, 1821) & Pica-pau-de-asa-vermelha & $\mathrm{OB}, \mathrm{V}, \mathrm{CAP}$ & 1 \\
\hline \multicolumn{4}{|l|}{ Thamnophilidae } \\
\hline Taraba major (Vieillot, 1816) & Cancão-de-fogo & $\mathrm{OB}, \mathrm{V}$ & 1 \\
\hline Thamnophilus doliatus (Linnaeus, 1764) & Choca-barrada & $\mathrm{OB}, \mathrm{V}$ & 1 \\
\hline Thamnophilus palliatus (Lichtenstein, 1823) & Choca-listrada & $\mathrm{OB}, \mathrm{V}$ & 1 \\
\hline Thamnophilus caerulescens Vieillot, 1816 & Espanta-raposa & V & 1 \\
\hline Thamnophilus aethiops Sclater, 1858 & Choca-lisa & $\mathrm{OB}, \mathrm{V}, \mathrm{CAP}$ & 1 \\
\hline
\end{tabular}


Tabela I. Continuação.

\begin{tabular}{|c|c|c|c|}
\hline Família/Espécie & Nome popular & Registro & Dieta \\
\hline Dysithamnus mentalis (Temminck, 1823) & Choquinha-lisa & $\mathrm{OB}, \mathrm{V}$ & I \\
\hline Thamnomanes caesius (Temminck, 1820) & Ipecuá & $\mathrm{OB}, \mathrm{V}$ & I \\
\hline Myrmotherula axillaris Vieillot, 1817 & Choquinha-de-flancos-brancos & $\mathrm{OB}, \mathrm{V}, \mathrm{CAP}$ & I \\
\hline Herpsilochmus rufimarginatus (Temminck, 1822) & Chorozinho-de-asa-ruiva & $\mathrm{OB}, \mathrm{V}$ & I \\
\hline Herpsilochmus atricapillus Pelzeln, 1868 & Chorozinho-de-chapeu-preto & $\mathrm{OB}, \mathrm{V}$ & 1 \\
\hline Formicivora grisea (Boddaert, 1783) & Papa-formigas-pardo & $\mathrm{OB}, \mathrm{V}$ & I \\
\hline Pyriglena leuconota (Spix, 1824) & Papa-taoca & CAP & 1 \\
\hline Myrmeciza ruficauda (Wied, 1831) & Papa-formigas-de-cauda-ruiva & V & I \\
\hline \multicolumn{4}{|l|}{ Conopophagidae } \\
\hline Conopophaga melanops (Wied, 1831) & Chupa-dente-de-máscara-preta & CAP & 1 \\
\hline Conopophaga lineata (Vieillot, 1818) & Chupa-dente & CAP & 1 \\
\hline \multicolumn{4}{|l|}{ Furnariidae } \\
\hline \multicolumn{4}{|l|}{ Furnariinae } \\
\hline Furnarius leucopus Swainson, 1838 & Amassa-barro & $\mathrm{OB}, \mathrm{V}$ & 1 \\
\hline \multicolumn{4}{|l|}{ Synallaxinae } \\
\hline Synallaxis frontalis Pelzeln, 1859 & Tio-antônio & $\mathrm{OB}, \mathrm{V}$ & I \\
\hline Synallaxis scutata Sclater, 1859 & Estrelinha-preta & $\mathrm{OB}, \mathrm{V}$ & 1 \\
\hline Certhiaxis cinnamomeus (Gmelin, 1788) & Casaca-de-couro & $\mathrm{OB}, \mathrm{V}$ & 1 \\
\hline Phacellodomus rufifrons (Wied, 1821) & Ferreiro & $\mathrm{OB}, \mathrm{V}$ & 1 \\
\hline \multicolumn{4}{|l|}{ Philydorinae } \\
\hline Xenops minutus (Sparrman, 1788) & Bico-virado-miúdo & $\mathrm{OB}, \mathrm{V}$ & 1 \\
\hline Xenops rutilans Temminck, 1821 & Bico-virado-carijó & $\mathrm{OB}, \mathrm{V}$ & 1 \\
\hline \multicolumn{4}{|l|}{ Dendrocolaptidae } \\
\hline Sittasomus griseicapillus (Vieillot, 1818) & Arapaçu-verde & $\mathrm{OB}, \mathrm{V}$ & 1 \\
\hline Xiphorhynchus picus (Gmelin, 1788) & Arapaçu-de-bico-branco & $\mathrm{OB}, \mathrm{V}$ & 1 \\
\hline Lepidocolaptes angustirostris (Vieillot, 1918) & Arapaçu-do-cerrado & $\mathrm{OB}, \mathrm{V}$ & 1 \\
\hline Xiphorhynchus fuscus (Vieillot, 1818) & Arapaçu-rajado & $\mathrm{OB}, \mathrm{V}, \mathrm{CAP}$ & 1 \\
\hline \multicolumn{4}{|l|}{ Tyrannidae } \\
\hline \multicolumn{4}{|l|}{ Elaeniinae } \\
\hline Zimmerius gracilipes (Sclater \& Salvin, 1867) & Poiaeiro-de-sobrancelha & $\mathrm{OB}, \mathrm{V}$ & 1 \\
\hline Camptostoma obsoletum (Temminck, 1824) & Risadinha & $\mathrm{OB}, \mathrm{V}$ & I \\
\hline Phaeomyias murina (Spix, 1825) & Bagageiro & $\mathrm{OB}, \mathrm{V}$ & $\mathrm{O}$ \\
\hline Myiopagis viridicata (Vieillot, 1817) & Guaracava-esverdeada & $\mathrm{OB}, \mathrm{V}$ & 1 \\
\hline Myiopagis gaimardii (d'Orbigny, 1839) & Maria-pechim & V & I \\
\hline Elaenia flavogaster (Thunberg, 1822) & Maria-já-é-dia & $\mathrm{OB}, \mathrm{V}$ & $\mathrm{O}$ \\
\hline Elaenia spectabilis (Pelzeln, 1868) & Guaracava-grande & $\mathrm{OB}, \mathrm{V}$ & $\mathrm{O}$ \\
\hline Elaenia mesoleuca (Cabanis \& Heine, 1859) & Gordinho & $\mathrm{OB}, \mathrm{V}$ & $\mathrm{O}$ \\
\hline Elaenia cristata (Pelzeln, 1868) & Papa-enxeico & OB & $\mathrm{O}$ \\
\hline Mionectes oleagineus (Lichtenstein, 1823) & Abre-asas & $\mathrm{OB}, \mathrm{V}, \mathrm{CAP}$ & $\mathrm{O}$ \\
\hline Leptopogon amaurocephalus Tschudi, 1846 & Cabeçudo & $\mathrm{OB}, \mathrm{V}$ & 1 \\
\hline Capsiempis flaveola (Lichtenstein, 1823) & Marianinha & $\mathrm{OB}, \mathrm{V}$ & I \\
\hline Hemitriccus margaritaceiventer (Lafresnaye \& d'Orbigny, 1837) & Sebinho-olho-de-ouro & $\mathrm{OB}, \mathrm{V}$ & I \\
\hline Hemitriccus zosterops (Pelzeln, 1868) & Maria-de-olho-branco & $\mathrm{OB}, \mathrm{V}, \mathrm{CAP}$ & 1 \\
\hline Todirostrum cinereum (Linnaeus, 1766) & Reloginho & $\mathrm{OB}, \mathrm{V}$ & 1 \\
\hline
\end{tabular}


Tabela I. Continuação.

\begin{tabular}{|c|c|c|c|}
\hline Família/Espécie & Nome popular & Registro & Dieta \\
\hline Poecilotriccus fumifrons (Hartlaub, 1853) & Ferreirinho & $\mathrm{OB}, \mathrm{V}$ & 1 \\
\hline Rhynchocyclus olivaceus (Temminck, 1820) & Bico-chato-oliváceo & $\mathrm{OB}, \mathrm{V}, \mathrm{CAP}$ & 1 \\
\hline Tolmomyias sulphurescens (Spix, 1825) & Bico-chato-de-orelha-preta & OB & 1 \\
\hline Tolmomyias poliocephalus (Taszanowski, 1884) & Bico-chato-de-cabeça-cinza & $\mathrm{OB}, \mathrm{V}$ & I \\
\hline Tolmomyias flaviventris (Wied, 1831) & Bico-chato-amarelo & $\mathrm{OB}, \mathrm{V}, \mathrm{CAP}$ & 1 \\
\hline Platyrinchus mystaceus Vieillot, 1818 & Patinho & $\mathrm{CP}$ & 1 \\
\hline \multicolumn{4}{|l|}{ Fluvicolinae } \\
\hline Myiobius barbatus (Gmelin, 1789) & Assanhadinho & $\mathrm{OB}, \mathrm{V}, \mathrm{CAP}$ & 1 \\
\hline Myiophobus fasciatus (Temminck, 1822) & Felipe & $\mathrm{OB}, \mathrm{V}$ & I \\
\hline Lathrotriccus euleri (Cabanis, 1868) & Enferrujado & $\mathrm{OB}, \mathrm{V}$ & 1 \\
\hline Cnemotriccus fuscatus (Wied, 1831) & Guaracavuçu & $\mathrm{OB}, \mathrm{V}$ & 1 \\
\hline Fluvicola nengeta (Vieillot, 1824) & Lavandeira & OB & I \\
\hline Arundinicola leucocephala (Linnaeus, 1764) & Viuvinha & OB & I \\
\hline Machetornis rixosa (Vieillot, 1819) & Bem-te-vi-do-gado & $\mathrm{OB}, \mathrm{V}$ & 1 \\
\hline \multicolumn{4}{|l|}{ Tyranninae } \\
\hline Rhytipterna simplex (Lichtenstein, 1823) & Planadeira & $\mathrm{OB}, \mathrm{V}$ & I \\
\hline Myiarchus ferox (Gmelin, 1789) & Mané-besta & $\mathrm{OB}, \mathrm{V}$ & I \\
\hline Myiarchus tyrannulus (Müller, 1776) & Mané-besta & $\mathrm{OB}, \mathrm{V}$ & 1 \\
\hline Myiarchus swainsoni (Berlepsch, 1883) & Irrê & $\mathrm{OB}, \mathrm{V}$ & 1 \\
\hline Myiarchus tuberculifer (Lafresnaye \& d'Orbigny, 1837) & Maria-cavaleira-pequena & $\mathrm{OB}, \mathrm{V}$ & I \\
\hline Pitangus sulphuratus (Linnaeus, 1766) & Bem-te-vi & $\mathrm{OB}, \mathrm{V}$ & $\mathrm{O}$ \\
\hline Megarynchus pitangua (Linnaeus, 1766) & Bem-te-vi-de-bico-de-gamela & $\mathrm{OB}, \mathrm{V}$ & $\mathrm{O}$ \\
\hline Myiozetetes similis (Spix, 1825) & Bem-te-vizinho-de-coroa-vermelha & $\mathrm{OB}, \mathrm{V}$ & $\mathrm{O}$ \\
\hline Myiodynastes maculatus (Müller, 1776) & Bem-te-vi-rajado & $\mathrm{OB}, \mathrm{V}$ & $\mathrm{O}$ \\
\hline Legatus leucophaius (Vieillot, 1818) & Bem-te-vi-pirata & $\mathrm{OB}, \mathrm{V}$ & 1 \\
\hline Empidonomus varius (Vieillot, 1818) & Bem-te-vi-peitica & $\mathrm{OB}, \mathrm{V}$ & I \\
\hline Tyrannus melancholicus Vieillot, 1819 & Suiriri & $\mathrm{OB}, \mathrm{V}$ & I \\
\hline \multicolumn{4}{|l|}{ Tityrinae } \\
\hline Pachyramphus viridis (Vieillot, 1816) & Caneleiro-verde & OB & I \\
\hline Pachyramphus polychopterus (Vieillot, 1818) & Caneleiro-preto & $\mathrm{OB}, \mathrm{V}$ & I \\
\hline Pachyramphus validus (Vieillot, 1816) & Caneleiro-de-chapeu-preto & OB & I \\
\hline \multicolumn{4}{|l|}{ Pipridae } \\
\hline Pipra rubrocapilla Temminck, 1821 & Cabeça-encarnada & $\mathrm{OB}, \mathrm{V}, \mathrm{CAP}$ & $\mathrm{F}$ \\
\hline Chiroxiphia pareola (Linnaeus, 1766) & Dançarino & $\mathrm{OB}, \mathrm{V}, \mathrm{CAP}$ & $\mathrm{F}$ \\
\hline Manacus manacus (Linnaeus, 1766) & Rendeira & OB,V,CAP & $\mathrm{F}$ \\
\hline Neopelma pallescens (Lafresnaye, 1853) & Fruxu & $\mathrm{OB}, \mathrm{V}, \mathrm{CAP}$ & $\mathrm{O}$ \\
\hline Schiffornis turdina (Wied, 1831) & Flautim-marrom & $\mathrm{OB}, \mathrm{V}$ & $\mathrm{F}$ \\
\hline \multicolumn{4}{|l|}{ Hirundinidae } \\
\hline Tachycineta albiventer (Boddaert, 1783) & Andorinha-do-rio & $\mathrm{OB}$ & 1 \\
\hline Progne chalybea (Gmelin, 1789) & Andorinha-doméstica-grande & OB & 1 \\
\hline Alopochelidon fucata (Temminck, 1822) & Andorinha-morena & OB & I \\
\hline Stelgidopteryx ruficollis (Vieillot, 1817) & Andorinha-serrador & OB & 1 \\
\hline Hirundo rustica (Boddaert, 1783) & Andorinha-de-bando & OB & 1 \\
\hline
\end{tabular}


Tabela I. Continuação.

\begin{tabular}{|c|c|c|c|}
\hline Família/Espécie & Nome popular & Registro & Dieta \\
\hline \multicolumn{4}{|l|}{ Troglodytidae } \\
\hline Donacobius atricapilla (Linnaeus, 1766) & Chaú & $\mathrm{OB}, \mathrm{V}$ & I \\
\hline Thryothorus genibarbis Swainson, 1837 & Garrinchão-pai-avô & $\mathrm{OB}, \mathrm{V}$ & 1 \\
\hline Troglodytes musculus Naumann, 1823 & Rouxinol & $\mathrm{OB}, \mathrm{V}$ & 1 \\
\hline \multicolumn{4}{|l|}{ Muscicapidae } \\
\hline \multicolumn{4}{|l|}{ Sylviinae } \\
\hline Ramphocaenus melanurus Vieillot, 1819 & Bico-assovelado & $\mathrm{OB}, \mathrm{V}$ & 1 \\
\hline Polioptila plumbea (Gmelin, 1788) & Rabo-mole & $\mathrm{OB}, \mathrm{V}$ & 1 \\
\hline \multicolumn{4}{|l|}{ Turdinae } \\
\hline Turdus rufiventris Vieillot, 1818 & Sabiá-gongá & $\mathrm{OB}, \mathrm{V}, \mathrm{CAP}$ & $\mathrm{O}$ \\
\hline Turdus leucomelas Vieillot, 1818 & Sabiá-branco & $\mathrm{OB}, \mathrm{V}, \mathrm{CAP}$ & $\mathrm{O}$ \\
\hline Turdus amaurochalinus Cabanis, 1851 & Sabiá-bico-de-osso & OB & $\mathrm{O}$ \\
\hline Turdus fumigatus Lichtenstein, 1823 & Sabiá-da-mata & OB & $\mathrm{O}$ \\
\hline \multicolumn{4}{|l|}{ Vireonidae } \\
\hline Cyclarhis gujanensis (Gmelin, 1789) & Pitiguari & $\mathrm{OB}, \mathrm{V}$ & 1 \\
\hline Vireo olivaceus (Vieillot, 1817) & Juruviara & $\mathrm{OB}, \mathrm{V}$ & 1 \\
\hline Hylophilus amaurocephalus (Nordmann, 1835) & Verdinho-coroado & $\mathrm{OB}, \mathrm{V}, \mathrm{CAP}$ & 1 \\
\hline \multicolumn{4}{|l|}{ Emberizidae } \\
\hline \multicolumn{4}{|l|}{ Parulinae } \\
\hline Basileuterus flaveolus (Baird, 1865) & Canário-do-mato & $\mathrm{OB}, \mathrm{V}$ & I \\
\hline Basileuterus culicivorus (Lichtenstein, 1830) & Pula-pula & $\mathrm{OB}, \mathrm{V}$ & $\mathrm{I}$ \\
\hline \multicolumn{4}{|l|}{ Coerebinae } \\
\hline Coereba flaveola (Linnaeus, 1758) & Sebito & $\mathrm{OB}, \mathrm{V}$ & $\mathrm{O}$ \\
\hline \multicolumn{4}{|l|}{ Thraupinae } \\
\hline Cissopis leveriana (Gmelin, 1788) & Pêga & OB & $\mathrm{O}$ \\
\hline Thlypopsis sordida (Lafresnaye \& d'Orbigny, 1837) & Canário-de-folha & OB & $\mathrm{O}$ \\
\hline Nemosia pileata (Boddaert, 1783) & Saíra-de-chapéu-preto & $\mathrm{OB}, \mathrm{V}$ & $\mathrm{O}$ \\
\hline Tachyphonus cristatus (Linnaeus, 1766) & Tiê-galo & $\mathrm{OB}, \mathrm{V}$ & $\mathrm{O}$ \\
\hline Tachyphonus rufus (Boddaert, 1783) & Encontro-de-prata & $\mathrm{OB}, \mathrm{V}$ & $\mathrm{O}$ \\
\hline Ramphocelus bresilius (Linnaeus, 1766) & Sague-de-boi & $\mathrm{OB}, \mathrm{V}$ & $\mathrm{O}$ \\
\hline Thraupis sayaca (Linnaeus, 1766) & Sanhaçu-de-bananeira & $\mathrm{OB}, \mathrm{V}$ & $\mathrm{O}$ \\
\hline Thraupis palmarum (Wied, 1821) & Sanhaçu-de-coqueiro & $\mathrm{OB}, \mathrm{V}$ & $\mathrm{O}$ \\
\hline Euphonia chlorotica (Linnaeus, 1776) & Vem-vem & $\mathrm{OB}, \mathrm{V}$ & $\mathrm{O}$ \\
\hline Euphonia violacea (Linnaeus, 1758) & Guriatã & $\mathrm{OB}, \mathrm{V}$ & $\mathrm{F}$ \\
\hline Tangara fastuosa (Lesson, 1831) & Pintor-verdadeiro & OB & $\mathrm{F}$ \\
\hline Tangara cyanocephala (Müller, 1766) & Pintor-mirim & OB & $\mathrm{O}$ \\
\hline Tangara cayana (Linnaeus, 1766) & Frei-vicente & $\mathrm{OB}, \mathrm{V}$ & $\mathrm{O}$ \\
\hline Tangara velia (Wied, 1830) & Pintor-estrela & OB & $\mathrm{F}$ \\
\hline Dacnis cayana (Linnaeus, 1766) & Saí-azul & OB & $\mathrm{O}$ \\
\hline Cyanerpes cyaneus (Linnaeus, 1766) & Saíra-beija-flor & OB & $\mathrm{F}$ \\
\hline \multicolumn{4}{|l|}{ Emberizinae } \\
\hline Sicalis luteola (Sparrman, 1789) & Mané-mago & EN & G \\
\hline Volatinia jacarina (Linnaeus, 1766) & Tiziu & $\mathrm{OB}, \mathrm{V}$ & G \\
\hline Sporophila lineola (Linnaeus, 1758) & Bigode & $\mathrm{OB}, \mathrm{V}$ & G \\
\hline
\end{tabular}


Tabela I. Continuação.

\begin{tabular}{|c|c|c|c|}
\hline Família/Espécie & Nome popular & Registro & Dieta \\
\hline Sporophila nigricollis (Vieillot, 1823) & Papa-capim & $\mathrm{OB}, \mathrm{V}$ & G \\
\hline Sporophila albogularis (Spix, 1825) & Patativa-golada & EN & G \\
\hline Sporophila leucoptera (Vieillot, 1817) & Chorão & OB & G \\
\hline Sporophila bouvreuil (Sclater, 1864) & Caboclinho & OB & G \\
\hline Tiaris fuliginosus (Wied, 1830) & Cigarra-de-coqueiro & OB & G \\
\hline Arremon taciturnus (Hermann, 1783) & Tico-tico-da-mata & $\mathrm{OB}, \mathrm{V}$ & $\mathrm{O}$ \\
\hline Paroaria dominicana (Linnaeus, 1758) & Galo-de-campina & OB & G \\
\hline \multicolumn{4}{|l|}{ Cardialinae } \\
\hline Saltator maximus (Müller, 1776) & Trinca-ferro & $\mathrm{OB}, \mathrm{V}, \mathrm{CAP}$ & $\mathrm{O}$ \\
\hline \multicolumn{4}{|l|}{ Icterinae } \\
\hline Cacicus solitarius (Vieillot, 1816) & Bauá & OB & $\mathrm{O}$ \\
\hline Icterus cayanensis (Linnaeus, 1766) & Encontro-de-ouro & $\mathrm{OB}, \mathrm{V}$ & $\mathrm{O}$ \\
\hline Icterus jamacaii (Gmelin, 1788) & Concriz & $\mathrm{OB}, \mathrm{V}$ & $\mathrm{F}$ \\
\hline Leistes superciliaris (Bonaparte, 1850) & Papo-de-fogo & $\mathrm{OB}, \mathrm{V}$ & $\mathrm{O}$ \\
\hline Curaeus forbesi (Sclater, 1886) & Papa-arroz & $\mathrm{OB}, \mathrm{V}$ & $\mathrm{O}$ \\
\hline Molothrus bonariensis (Gmelin, 1789) & Pássaro-preto & $\mathrm{OB}$ & $\mathrm{O}$ \\
\hline \multicolumn{4}{|l|}{ Passeridae } \\
\hline Passer domesticus (Linnaeus, 1758) & Pardal & $\mathrm{OB}, \mathrm{V}$ & $\mathrm{O}$ \\
\hline \multicolumn{4}{|l|}{ Estrildidae } \\
\hline Estrilda astrild (Vieillot, 1805) & Bico-de-lacre & $\mathrm{OB}, \mathrm{V}$ & G \\
\hline
\end{tabular}

Tabela II. Distribuição das guildas das espécies registradas na Reserva Estadual de Gurjaú, Cabo de Santo Agostinho, Pernambuco.

\begin{tabular}{lcc}
\hline \multirow{2}{*}{ Tipo de dieta } & \multicolumn{2}{c}{ Proporções de espécies por categoria trófica } \\
\cline { 2 - 3 } Carnívoro & $\mathrm{N}$ & Porcentagem (\%) \\
\hline Detritívoro & 10 & 4,5 \\
Frugívoro & 3 & 1,3 \\
Granívoro & 18 & 8,0 \\
Insetívoro & 16 & 7,1 \\
Nectarívoro & 99 & 44,0 \\
Onívoro & 16 & 7,1 \\
Piscívoro & 59 & 26,2 \\
\hline Total & 4 & 1,8 \\
\hline
\end{tabular}

e Pulsatrix perspicillata (Latham, 1790) como foi observado por Lyra-Neves et al. (2004). A presença dessas aves pode ser explicada, por encontrarem na RESG uma área propícia para o seu refúgio e a aquisição do alimento. De acordo com Aleixo (1999), os carnívoros de grande porte podem ser prejudicados pela necessidade de uma grande área de vida e por alteração na abundância de suas presas.
Com os menores percentuais, os detritívoros e piscívoros foram representados pelos Cathartidae e Alcedinidae. Os Cathartidae foram avistados sobrevoando ou alimentando-se de animais em decomposição dentro ou nos arredores da RESG. Os Alcedinidae foram observados forrageando nos riachos e açudes internos ou no entorno da RESG. Este baixo percentual segue o padrão encontrado em outras áreas estudadas (eg. WiLlis 1979, MotTa-Júnior 1990, Anjos 1998, Aleixo 1999, Anjos \& Bóçon 1999, Dário \& Almeida 2000, Krügel \& Anjos 2000, Anjos 2001, Blamires et al. 2001, SANtos 2001, Silveira et al. 2003, Efe 2001).

Foram registradas para a RESG as seguintes espécies de aves seguidores de correição e insetívoros de subosque: Pyriglena leuconota (Spix, 1824), Conopophaga melanops (Wied, 1831), Platyrinchus mystaceus Vieillot, 1818 e Myiobius barbatus (Gmelin, 1789) (SICK 1997). Estas aves não foram facilmente observadas ou capturadas. Este fato pode ter ocorrido devido à redução do número de indivíduos destas espécies, por habitarem fragmentos parcialmente alterados por monoculturas de subsistência, ocasionando assim o aumento do efeito de borda.

Trabalhos realizados por STOUfFer \& BierRegaARd Jr. (1995a) relatam o desaparecimento de aves insetívoras de subosque, principalmente as seguidoras de formigas de correição como $P$. leuconota, que são as primeiras a desaparecer (BIERREGAARD JR \& Lovejoy 1989). Segundo Askins et al. (1987) e Stouffer \& BierRegaARd JR. (1995b), espécies dependentes de interior de floresta desaparecem em fragmentos de mata pequenos, portanto elas são vul- 
neráveis à fragmentação que aumenta o efeito de borda.

Em Gurjaú, foram observadas espécies insetívoras especialistas, seguidoras de correição e de frugívoros de grande porte. As espécies dessas categorias tróficas são bastante vulneráveis e podem ser eliminadas de áreas com um acentuado efeito de borda devido à fragmentação (Willis 1979, JANZEN 1983, BiERREGAARD Jr. et al. 1992, ANDrÉn 1994, Anjos 1998, Silva \& TABARelli 2000). Os resultados deste trabalho mostram que a RESG, ainda mantém espécies de insetívoros especialistas como: Pyriglena leuconota, Conopophaga melanops e C. lineata, e de grandes frugívoros como: Pionus maximiliani, Pteroglossus aracari e P. inscriptus.

A estrutura trófica da comunidade avifaunística da Reserva Estadual de Gurjaú, ainda se apresenta equilibrada, com presença de espécies de aves como: insetívoros especialistas dependentes de interior de floresta, predadores de topo de cadeia e grandes frugívoros. Assim, a conservação da Reserva Ecológica de Gurjaú deve ser mantida pelo órgão responsável por sua guarda, firmando parcerias com órgãos de fiscalização do meio ambiente do estado. Desta forma espera-se diminuir a caça, a queima e a retirada ilegal de madeira no período do corte da cana, realizada pelos usineiros com intuito de aumentar a área de plantio e pelos moradores que retiram e comercializam ilegalmente a madeira, resultando na diminuição do fragmento, com perdas da biodiversidade da área.

\section{AGRADECIMENTOS}

Agradecemos à Coordenação de Aperfeiçoamento de Pessoal de Nível Superior (CAPES) pela bolsa de Doutorado de Wallace Rodrigues Telino Júnior e Rachel Maria de Lyra Neves, à Fundação Apolônio Sales de Apoio e Desenvolvimento à Pesquisa (FADURPE), ao Departamento de Estradas e Rodagens de Pernambuco (DER/PE), à Companhia Pernambucana de Meio Ambiente de Pernambuco $(\mathrm{CPRH})$ e à Companhia Pernambucana de abastecimento de Água (COMPESA), pelo suporte dado no decorrer desta pesquisa.

\section{REFERÊNCIAS BIBLIOGRÁFICAS}

AleiXo, A. 1999. Effects of selective logging on a bird community in the Brazilian Atlantic Forest. The Condor, Camarillo, 101: 537-548.

Aleixo, A. \& J.M.E. Vielliard. 1995. Composição e dinâmica da avifauna da Mata de Santa Genebra, Campinas, São Paulo, Brasil. Revista Brasileira de Zoologia, Curitiba, 12 (3): 493511.

Aleixo, A. \& M. Galetti. 1997. The conservation of the avifauna in a lowland Atlantic Forest in South-east Brazil. Bird Conservation International, Cambridge, 7: 235-261.

AlmeidA, A.F. 1982. Análise das categorias de nichos tróficos das aves de matas ciliares em Anhembi, Estado de São Paulo. Silvicultura em São Paulo, São Paulo, 16 (3): 1787-795.

AndréN, H. 1994. Effects of habitat fragmentation on birds and mammals in landscapes with different proportions of suitable habitat: a review. Oikos, Copenhagen, 71: 355-366. ANjos, L. Dos. 1998. Conseqüências biológicas da fragmentação no norte do Paraná. IPEF, Piracicaba, 12 (32): 87-94.

Anjos, L. Dos. 2001. Bird Communities in five Atlantic Forest fragments in Southern Brazil. Ornitologia Neotropical, St. Louis, 12: 11-27.

Anjos, L. Dos \&. R. Bóçon. 1999. Bird communities in natural forest patches in southern Brazil. The Wilson Bulletin, Lawrence, 111 (3): 397-414.

Askins, R.A.; M.J. Philbrick \& D.S. Sugeno. 1987. Relationship between the regional abundance of forest and the composition of forest bird communities. Biological Conservation, Davis, 39: 129-152.

Blamires, D.; A.B. Valgas \& P. DA D. Bispo. 2001. Estrutura da comunidade de aves da Fazenda Bonsucesso, município de Caldazinha, Goiás, Brasil. Tangara, Belo Horizonte, 1 (3): 101-113.

Bierregatrd JR., R.O. \& T.E. Lovejoy. 1989. Effects of forest fragmentation on Amazonian understory bird communities. Acta Amazônica, Manaus, 19: 215-241.

Bierregaard Jr., R. O.; T. E. Lovejoy; V. Kapos; A. A. dos Santos \& R. W. Hutchings. 1992. The biological dynamics of tropical rainforest fragments: a prospective comparison of fragments and continuous forest. BioScience, Washington, 42 (11): 859-866.

Brooks, T.M.; R.A. Mittermeier; C.G. Mittermeier; G.A.B. DA Fonseca; A.B. Rylands; W.R. Konstant; P. Flick; J. Pilgrim; S. Oldfield; G. Mangin \& C. Hilton-Taylor. 2002. Habitat loss and extinction in the hotspots of biodiversity. Conservation Biology, Montpelier, 16 (4): 909-920.

DÁrio, F.R. \& A.F. DE Almeida. 2000. Influência de corredor florestal sobre a avifauna de Mata Atlântica. Scientia Forestalis, Piracicaba, 58: 99-109.

D’Angelo-Neto, S.; N. Venturin; A.T. de Oliveira-Filho \& F.A.F. Costa. 1998. Avifauna de quatro fisionomias florestais de pequeno tamanho (5-8 ha) no Campus da UFLA. Revista Brasileira de Biologia, Rio de Janeiro, 58 (3): 463-472.

Donatelli, R.J.; T.V.V. DA Costa \& C.D. Ferreira. 2004. Dinâmica da avifauna em fragmento de mata na Fazenda Rio Claro, Lençóis Paulista, São Paulo, Brasil. Revista Brasileira de Zoologia, Curitiba, 21 (1): 97-114.

Dossiê Mata Atlântica. 2001. Monitoramento Participativo da Mata Atlântica. São Paulo, Ipsis Gráfica e Editora, 409p.

EFE, M. 2001. Inventário e distribuição a avifauna do Parque Saint' Hilaire, Viamão, Rio Grande do Sul, Brasil. Tangara, Belo Horizonte, 1 (1): 12-25.

Farias, G.B.; G.L. Pacheco \& M.T. de Brito. 2000. Aves de Pernambuco e seus nomes populares. Recife, Editora Universitária Universidade Federal de Pernambuco, 55p.

FIDEM. 1987. Região Metropolitana do Recife: Reservas Ecológicas. Recife, Governo do Estado de Pernambuco, Secretaria de Planejamento do Estado de Pernambuco, Fundação de Desenvolvimento da Região Metropolitana do Recife, I+108p. 
Guilherme, E. 2001. Comunidade de aves do Campus e Parque Zoobotânico da Universidade Federal do Acre, Brasil. Tangara, Belo Horizonte, 1 (2): 57-73.

Hempel, A. 1949. Estudo da alimentação natural de aves silvestres do Brasil. Arquivos do Instituto Biológico, São Paulo, 19: 237-268.

JANZEN, D.H. 1983. No park is na island: increase interference from outside as park size decreases. Oikos, Copenhagen, 41: 402-410.

Jiмво, S. 1957. A flora na alimentação das aves brasileira: II Alimentação da codorna (Nothura maculosa maculosa). Papéis Avulsos Zoologia, São Paulo, 13 (8): 99-108.

JonHs, A.D. 1991. Responses of Amazonian Rain Forest birds to habitat modification. Journal of Tropical Ecology, Winchelsea, 7: 417-437.

KARR, J.R. \& R.R. Rотн. 1971. Vegetation structure and avian diversity in several new world areas. The American Naturalist, Chicago, 105: 423-435.

KrüGuel, M.M. \& L. Dos Anjos. 2000. Bird communities in Forest remnants in the city of Maringá, Paraná state, southern Brazil. Ornitologia Neotropical, Montreal, 11: 315-330.

Kuhlmann, M. \& S. Jimbo. 1957. A flora na alimentação das aves brasileira: I Generalidades. Papéis Avulsos Zoologia, São Paulo, 13 (7): 85-97.

Lyra-Neves, R.M. de; M.M. Dias; S.M. de Azevedo Júnior; W.R. Telino-Júnior \& M.E.L. De LaRRAZÁbal. 2004. Ecologia quantitativa das aves em um fragmento florestal na Zona da Mata Sul, no Estado de Pernambuco, Brasil. Revista Brasileira de Zoologia, Curitiba, 21 (3): 581-592.

MARINI, M.Â. 2000. Efeitos da fragmentação florestal sobre as aves em Minas Gerais, p. 41-54. In: M.A. Dos SANTos-Alves; J.M.C. da Silva; M. Van Sluys; H. De G. Bergallo \& C.F.D. DA Rосна (Eds). A ornitologia no Brasil: pesquisa atual e perspectivas. Rio de Janeiro, Editora Universidade do Estado do Rio de Janeiro, 352p.

Moojen, J.; J.C. de Carvalho \& H. DE S. Lopes. 1941. Observações sobre o conteúdo gástrico das aves brasileiras. Memórias do Instituto Oswaldo Cruz, Rio de Janeiro, 36 (3): 405-444.

MotTA-Júnior, J.C. 1990. Estrutura trófica e composição das avifaunas de três habitas terrestres na região central do Estado de São Paulo. Ararajuba, Rio de Janeiro, 1: 65-71.

Myers, N.; R.A. Mittermeier; C.G. Mittermeier; G.A.B. DA FonsecA \& J. Kent. 2000. Biodiversity hotspots for conservation priorities. Nature, London, 403: 853-858.

Pacheco, J.F. \& B.M. Whithey. 1995. Range extensions for some birds in northeastern Brazil. Bulletin of the British Ornithologists' Club, London, 115 (3): 157-163.

PinTo, O.M. DE O. 1940. Aves de Pernambuco. Breve ensaio retrospectivo com lista de exemplares coligidos e descrição de algumas formas novas. Arquivos de Zoologia do Estado de São Paulo, São Paulo, 1 (5): 219-282.

Piratelli, A. \& M.R. Pereira. 2002. Dieta das aves na região leste de Mato Grosso do Sul, Brasil. Ararajuba, Seropédica, 10 (2): 131-139.

Primack, R.B. \& E. Rodrigues. 2001. Biologia da Conservação. Londrina, E. Rodrigues, 328p.

Ranta, P.; T. Blom; J. Niemelã; E. Joensud \& M. Siitonen. 1998. The fragmented Atlantic Rain Forest of Brazil: size, shape and distribution of forest fragments. Biodiversity and Conservation, Dordrecht, 7: 385-403.

SANTOS, M.P.D. 2001. Composição da avifauna nas Áreas de Proteção Ambiental Serra da Tabatinga e Chapada das Mangabeiras, Brasil. Boletim do Museu Paraense Emílio Goeldi, Série Zoologia, Belém, 17 (1): 43-67.

Schubart, O.; A.C. Aguirre \& H. Sick. 1965. Contribuição para o conhecimento da alimentação das aves brasileiras. Arquivos de Zoologia, São Paulo, 12: 95-249.

SICK, H. 1997. Ornitologia brasileira. Rio de Janeiro, Nova Fronteira, II $+862 p$.

Silva, J.M.C. DA \& M. Tabarelli. 2000. Tree species impoverishment and the future flora of the Atlantic Forest of northeast Brazil. Nature, London, 404: 72-74.

Silveira, L.F.; F. Olmos \& A. Long. 2003. Birds in Atlantic Forest Fragments in northeastern, Brazil. Cotinga, Bedfordshire, 20: 32-46.

Stouffer, P.C. \& R.O. BierregaArd JR. 1995a. Effects of forest fragmentation on understory hummingbirds in Amazonian Brazil. Conservation Biology, Arlington, 9 (5): 1085-1094.

Stouffer, P.C. \& R.O. BierregaArd Jr. 1995b. Use of Amazonian forest fragments by understory insectivorous birds. Ecology, Durham, 76 (8): 2429-2445.

Terborgh, J. 1984. Habitat selection in Amazonian birds, p.311338. In: M.L. Cody (Ed.). Habitat selection in birds. Orlando, Academic Press, 558p.

Terborgh, J.; S.K. Robinson; T.A. Parker III; C.A. Munn \& N. Pierpont. 1990. Structure and organization of an Amazonian forest bird community. Ecological Monographs, Washington, 60 (2): 213-238.

Verner, J. 1981. Measuring responses of avian communities to habitat manipulation. Studies in Avian Biology, Los Angeles, 6: 543-547.

Willis, E.O. 1976. Effects of a cold wave on an Amazonian avifauna in the upper Paraguay drainage, western Mato Grosso, and suggestions on oscine-suboscine relationships. Acta Amazônica, Manaus, 6: 379-394.

Willis, E. O. 1979. The composition of avian communities in remanescent woodlots in southern Brazil. Papéis Avulsos de Zoologia, São Paulo, 33 (1): 1-25.

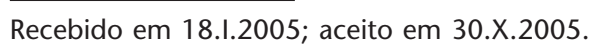

